\title{
Percepciones de teleformadores del Instituto de Formación y Estudios Sociales de Sevilla sobre la teleformación
}

\author{
Perceptions of teletrainers of the Institute of Learning and Social Studies of \\ Seville about teletraining
}

\author{
Bárbara Fernández Robles ${ }^{1}$, Julio Cabero Almenara ${ }^{2}$ \\ ${ }^{1}$ Universidad de Sevilla, España (bfernandezrobles@gmail.com) \\ ${ }^{2}$ Departamento Didáctica y Organización Escolar, Universidad de Sevilla, España (cabero@us.es)
}

Recibido el 7 de enero de 2016; revisado el 16 de febrero de 2016; aceptado el 29 de febrero de 2016; publicado el 1 de junio de 2016

\section{RESUMEN:}

En el presente artículo se analizan los factores que influyen en la formación de personas ocupadas impartida a través de teleformación, como resultado de una investigación que tenía como objetivo conocer las variables que hay que tener en cuenta en el diseño instruccional y en la puesta en marcha de las acciones formativas de personas ocupadas llevadas a cabo vía teleformación. El enfoque que se utilizó fue mixto, en concreto, los instrumentos que se utilizaron para la recolección de la información fueron cuestionario y entrevista. La muestra estaba compuesta por 20 teleformadores de personas ocupadas y por la coordinadora de los teleformadores del Instituto de Formación y Estudios Sociales de Sevilla. Los resultados apuntan que los encuestados perciben que la teleformación puede ser una acción válida para la formación de las personas ocupadas, dependiendo su éxito de prestarle atención a una serie de aspectos que van desde la forma de estructurar los cursos, hasta concebirlos de manera participativa y colaborativa.

PALABRAS CLAVE: APRENDIZAJE PERMANENTE, DESARROLLO PROFESIONAL, CURSOS EN LÍNEA, DISEÑO INSTRUCCIONAL, ESTRATEGIAS EDUCATIVAS.

\begin{abstract}
:
In this article we analyse the agents that influence in corporate training of employed people given by teletraining, as a result of a research which aim was to know all variables we have to take into account in instructional design and on setting up of the training activities of employed people carried out by teletraining. The focus we used was mixed, in particular, data collection instruments that we used were a questionnaire and an interview. The sample was composed by 20 teletrainers of employed people and by the teletrainer Coordinator of Institute of learning and Social Studies of Seville. The results suggest that teletraining could be an action valid for the training of employed people. Success depends on paying attention to a number of aspects such as how to structure the courses, or to conceive it as a participative and collaborative way.
\end{abstract}

KEYWORDS: LIFELONG LEARNING, PROFESSIONAL DEVELOPMENT, ONLINE COURSES, INSTRUCTIONAL DESIGN, EDUCATIONAL STRATEGIES. 


\section{INTRODUCCIÓN}

\subsection{La formación a lo largo de la vida: herramienta para mejorar la empleabilidad}

No es decir nada nuevo que las características de la Sociedad del Conocimiento (Cabero y Barroso, 2013) han tenido como consecuencia diferentes cambios que afectan al mundo laboral, modificando las competencias y perfiles profesionales, así como la desaparición y aparición de nuevos sectores productivos. Como consecuencia, los procesos de producción se modifican de forma constante, implicando cambios en la gestión del trabajo y apareciendo de esta forma nuevos servicios y requerimientos para los trabajadores (Rifkin, 2014, Vallejo, 2015).

A estos cambios, se une el actual panorama económico que influye de forma determinante en la estabilidad de los trabajadores; por este motivo, se necesita una mano de obra más formada $y$ cualificada (Blázquez, 2012; Sáez, 2010; Vallejo, 2015). Por lo señalado anteriormente, en la actualidad se está fomentando el aprendizaje a lo largo de la vida, debido a la toma de conciencia de la importancia que adquiere la renovación de conocimientos y competencias en momentos de cambio e incertidumbre (Fandos y Cano, 2013). Un reflejo claro de esta preocupación fue la denominación en 1996 por la Unión Europea el año de la formación a lo largo de la vida, para reclamar la necesidad de la formación permanente de la ciudadanía.

Al mismo tiempo, nos encontramos en una situación donde el aprendizaje no va a ser adquirido únicamente en contextos formales y en un período concreto de nuestra vida, sino que también se adquiere a través de los no formales e informales. Como señala Conner (2013), los contextos de aprendizaje en la sociedad del conocimiento van desde lo formal a lo informal, y desde lo intencional a lo inesperado.

Para los trabajadores supone un reto conciliar la vida personal y profesional con la formación, adquiriendo aquí un papel principal las nuevas tecnologías (Fandos y Cano, 2013; Sáez, 2010), ya que permiten flexibilizar los procesos de aprendizaje y la compatibilización de tiempos y espacios. Las TIC aplicadas a la formación y, en concreto, a la formación continua constituyen una herramienta de relevancia que puede aportar facilidades y valor añadido, contribuyendo a la mejora de la competitividad de los trabajadores y, por tanto, de las empresas en las que ejercen su labor profesional.

Es evidente, que la teleformación o e-learning ha surgido con fuerza para dar respuesta a las demandas formativas actuales de los trabajadores, puesto que permite compatibilizar las acciones formativas con el horario laboral, ya que como recalcan diferentes autores (Castaño, Maíz, Palacio y Villarroel, 2008; Cabero, 2013) se caracteriza por aportar flexibilidad en diferentes sentidos: temporal y espacial para la interacción de los participantes; para la selección de entre diferentes herramientas de comunicación, tanto sincrónicas como asincrónicas; para poder ofrecer la información a través de diferentes tipos de códigos; para poder elegir un itinerario específico formativo; o para apoyar el aprendizaje en comunidades de prácticas.

Las TIC facilitan el aprendizaje en red, permitiendo a las personas ocupadas compartir experiencia a la vez que van generando y reciclando el conocimiento, gracias al dialogo y la reflexión con otros. Como indica Carwile (2007) las nuevas tecnologías permiten al alumno construir el aprendizaje a través de la reflexión, gracias a que estas tecnologías facilitan interacciones de aprendizaje con los compañeros en línea, de manera que los alumnos adquieren una comprensión más profunda debido a las oportunidades de exposición de diferentes perspectivas e interpretaciones.

\subsection{Componentes básicos que garantizan el éxito en la teleformación}

Detrás de cualquier acción formativa existen una serie de factores que influyen de forma positiva o negativa en los logros de los objetivos. La planificación y el diseño de la instrucción son definitivas en todas las modalidades formativas, ya que es lo que permite pensar de manera coherente la secuencia de los aprendizajes. Pero se hace más imprescindible la rigurosidad de este diseño en la modalidad a distancia, debido a la separación maestro-alumno en tiempo y espacio (Martínez, 2009; Belloch, 2013).

El aprendizaje de personas ocupadas se caracteriza por ir destinado a personas adultas, característica fundamental para organizar la enseñanza, dado que los adultos cuentan con una experiencia y bagaje de vida que hay que tener en cuenta a la hora de diseñar la formación (Cabero y Castaño, 2007). Para Rodríguez (2014) algunos de los principios de los que se debe partir en el aprendizaje adulto: 
- Impulsar un aprendizaje generalizado, es decir, que el adulto educando sea capaz de aplicar el aprendizaje recibido en otras situaciones.

- Que el aprendizaje sea útil en dos sentidos: en utilidad y en aprender a aprender (que el aprendizaje facilite desenvolverse en nuevos aprendizajes).

- Atención individualizada.

- Facilitar un aprendizaje autónomo, por ejemplo el material y los ejercicios de autoevaluación deben ser de utilidad para facilitar este aprendizaje.

- Informar regularmente de su proceso de aprendizaje (p. 78).

Un aspecto importante y que no debe perderse de vista en las acciones formativas llevadas a cabo de forma telemática, es que el entorno virtual debe propiciar el trabajo colaborativo (Cabero, 2014; Núñez, 2011; Martín, Domínguez y Paralera, 2011; Martín y López, 2012;), ya que los alumnos de mayor edad se muestran más satisfechos cuando el aprendizaje es adquirido a través de la lectura de ideas y opiniones de compañeros. Dicha colaboración permite contrastar opiniones, favoreciendo la reflexión y la comunicación entre los participantes, a la vez que respeta la independencia y autonomía del alumno (Martín et ál., 2011).

No es nuestra intención analizar las ventajas que ofrece el e-learning a la teleformación, aspecto que ha sido analizado por diferentes autores (Monge, 2011; Cabero, 2014; Cuci, 2014; Vidal y Camaresa, 2014), y que van desde flexibilizar la formación, poder ofrecer una retroalimentación rápida $\mathrm{o}$ propiciar una formación apoyada en diferentes tipos de códigos. Ahora bien, si nos interesa señalar que la realización de acciones formativas de e-learning de calidad se consigue no olvidando que debemos alejarnos de las variables tecnológicas y centrarnos en las pedagógicas (Cabero, 2007; Cabero y Castaño, 2007), donde deberán centrarse en la estructuración de los contenidos (Cabero y Gisbert, 2005), las estrategias didácticas (Cabero y Pérez, 2007; Salinas, Pérez y De Benito, 2008), o el uso de diferentes herramientas de comunicación sincrónicas y asincrónicas (Shahabadi y Uplane, 2015).

Por su parte Tîrziu y Vrabieb (2015) nos señalan algunos factores que pueden dificultar la aplicación del e-learning y que son los siguientes:

- Apoyo y orientación para los estudiantes: hace referencia a los sistemas de apoyo necesarios para que un estudiante pueda seguir un curso en línea.
- Flexibilidad: tanto para seguir el curso como para llevar su propio ritmo de aprendizaje.

- Enseñanza, aprendizaje y actividades: lo que más afecta en el desempeño de los alumnos es la interactividad, el nivel de colaboración e interacción con sus compañeros.

- Acceso: calidad de la conexión.

- La experiencia académica de los estudiantes. La disposición del alumno a la enseñanza afecta en los resultados obtenidos.

- Localización de los contenidos, es decir, adaptación de los contenidos al entorno (costumbres, cultura...).

- Actitudes positivas o negativas provenientes del contexto (sociedad, políticos, estudiantes, profesores...).

\section{METODOLOGÍA Y DISEÑO DE INVESTIGACIÓN}

\section{Objetivo general.}

Conocer la valoración que realizan los profesionales de la teleformación sobre la implicación y repercusión de la teleformación en la formación de personas ocupadas.

En concreto, los objetivos específicos que se persiguen con la investigación son los siguientes:

- Valorar el impacto de la formación de personas ocupadas desarrollada vía teleformación.

- Analizar la metodología y medios didácticos que se deben utilizar en la formación de personas ocupadas impartida en la modalidad teleformación.

- Analizar la autonomía que deben tener los estudiantes en la teleformación.

- Apreciar las posibles desventajas del $e$ learning.

- Valorar las plataformas que se deben utilizar en la teleformación.

- Valorar la dificultad en el uso de las plataformas.

- Valorar el coste y el diseño de la formación a través de esta vía.

- Valorar si la teleformación logra igual o mejor preparación que los cursos impartidos de forma presencial.

- Valorar si hay relación entre el nivel formativo y las personas ocupadas que se forman vía teleformación. 


\subsection{Muestra}

En nuestra investigación hemos utilizado un tipo de muestreo intencional u opinático (Bisquerra, 2012), y estuvo conformada por 20 trabajadores de teleformación del Instituto de Formación y Estudios Sociales de Sevilla (IFES http://www.ifes.es/cursos/), más la coordinadora de la teleformación del centro de estudio.

En nuestro estudio hemos utilizado un enfoque mixto de investigación, en el cual seguimos las etapas que presentamos en la figura 1.

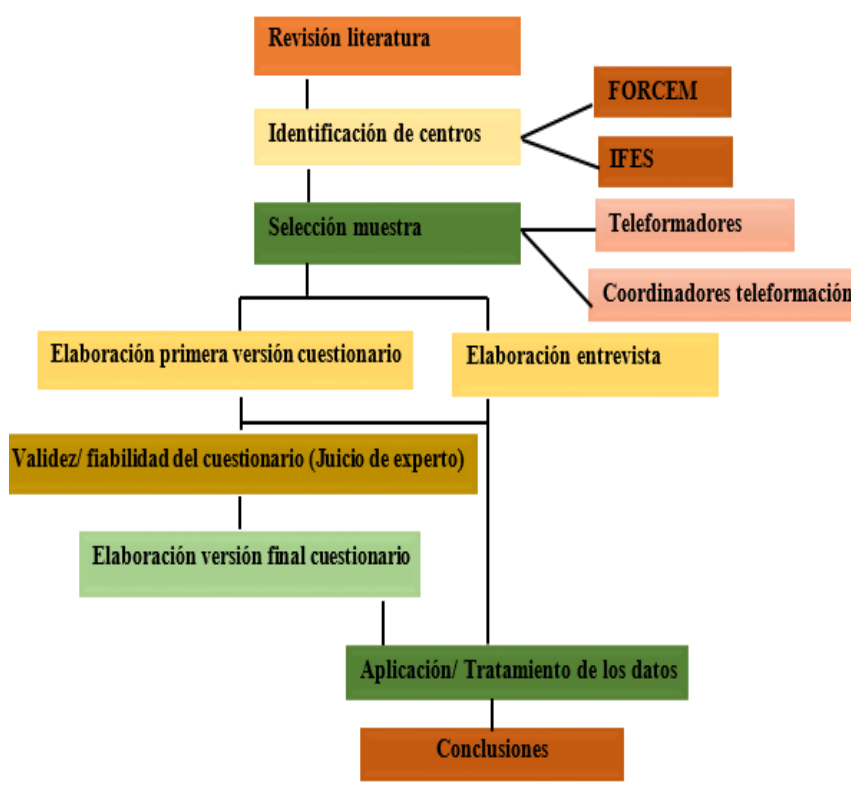

Figura 1. Fases de la investigación. Fuente: Elaboración propia.

Para la construcción del cuestionario y su validación manejamos la estrategia del juicio de expertos, utilizando los siguientes criterios para su selección:

- Haber impartido a lo largo de su vida profesional asignatura/contenidos/materias relacionadas con el terreno de la Tecnología Educativa, Nuevas Tecnologías Aplicadas a la Educación, formación a distancia o la formación virtual.

- Haber dirigido o participado en alguna investigación relacionada con aspectos de la formación virtual, formación a distancia, $e$ learning, entornos personales de aprendizaje.

- Haber realizado o participado en alguna publicación relacionada con aspectos de la formación virtual, formación a distancia, $e$ learning, entornos personales $\mathrm{de}$ aprendizaje.
Teniendo en cuenta los aspectos señalados anteriormente, contactamos con diferentes personas que pensábamos que podían responder a este perfil, y les invitamos a participar como expertos. Una vez que teníamos la muestra, diseñamos el juicio de experto, combinando el procedimiento del biograma con el de coeficiente de competencia experta, teniendo en cuenta las palabras de Barroso y Cabero (2013), donde destacan la importancia de combinar y utilizar ambos procedimientos para discriminar de una forma más adecuada la selección de los expertos. Por lo señalado y con la finalidad de corroborar que las personas seleccionadas eran las más adecuadas para nuestra investigación, planteamos cuestiones a los expertos para que valorasen su grado de conocimiento en el problema de investigación y respondiesen sobre su formación, labor profesional y experiencia profesional en el terreno de las tecnologías aplicadas a la educación. Tras este proceso de selección, contamos con ocho expertos para validar nuestro cuestionario.

Los expertos que validaron el cuestionario eran profesores que impartían asignaturas relacionadas con la Tecnología Educativa y la aplicación de las TIC en la educación de las universidades de Sevilla, Pablo Olavide y Alicante.

Una vez validado y reestructurado el cuestionario con las recomendaciones de los expertos, se diseñó su versión final que estuvo compuesta por 55 ítems. $\mathrm{Su}$ administración fue vía internet (https://docs.google.com/forms/d/1o09Upqw36PuvpJlykaHIlwRLb23mI1oQJBpNF6Qw8k/vie wform).

Señalar que también se llevó a cabo una entrevista a la coordinadora de teleformación de IFES, aunque en nuestro trabajo, para no extendernos solo presentaremos los datos referidos a los profesores de teleformación.

\section{RESULTADOS}

11 de los encuestados fueron hombres y 9 mujeres; la mayoría tenían entre 31 y 40 años $(\mathrm{f}=8)$, siguiéndole la etapa entre 41 y 50 años $(\mathrm{f}=7)$. Existiendo una minoría de teleformadores con edades comprendidas entre 25 y 30 años $(\mathrm{f}=3)$ y con más de 51 años $(\mathrm{f}=2)$.

Por lo que se refiere a su titulación, podemos destacar que hay variedad. No obstante, la rama educativa es la que mayor frecuencia adquiere $(\mathrm{f}=6)$. Coinciden las ciencias e ingenierías ( $\mathrm{f}=5$ ) y empresariales y economía ( $\mathrm{f}=5$ ), siendo los titulados en derecho los que menos frecuencia tienen $(\mathrm{f}=4)$. 
A continuación pasaremos a presentar los resultados alcanzados siguiendo las diferentes dimensiones que conformaban el instrumento.

\section{- Impacto de la teleformación}

Todos están de acuerdo en que la teleformación permite a las personas ocupadas actualizarse, facilitando su desarrollo profesional. Con respecto a si la teleformación permite el desarrollo personal, la mayoría se muestra muy de acuerdo o de acuerdo con esta afirmación ( $\mathrm{f}=18)$; no obstante, hay un mínimo de encuestados que se declaran moderadamente de acuerdo $(\mathrm{f}=1)$ y en desacuerdo $(\mathrm{f}=1)$.

Uno de los impactos que valoran positivamente es que la teleformación reduce el tiempo que las personas necesitan dedicar para llegar a ser y permanecer competentes en sus puestos de trabajo $(\mathrm{f}=14)$. Al mismo tiempo se muestran muy de acuerdo ( $\mathrm{f}=11$ ) o de acuerdo ( $\mathrm{f}=9$ ) con que con ella se aumenta la velocidad de poner en marcha acciones formativas. Estando al mismo tiempo de acuerdo $(\mathrm{f}=14)$ con que se consigue un aprendizaje significativo.

Para finalizar, señalar que se muestran muy de acuerdo $(\mathrm{f}=11)$ o de acuerdo $(\mathrm{f}=6)$ con que con la teleformación se pueden transferir conocimientos de calidad, aunque debemos destacar que hay 3 personas que están moderadamente de acuerdo.

\section{- Metodología y medios didácticos}

Todos están de acuerdo con que los contenidos deben estar adaptados a la modalidad telemática ( $f=19$ muy de acuerdo; $f=1$ de acuerdo), existiendo una combinación adecuada de contenidos teóricos y prácticos ( $\mathrm{f}=19$ muy de acuerdo; $\mathrm{f}=1$ de acuerdo). Resaltando también que los materiales y documentos disponibles deben ser claros, comprensibles y adecuados ( $\mathrm{f}=19$ muy de acuerdo; $\mathrm{f}=1$ de acuerdo).

Por lo que se refiere a la organización que deben tener los cursos existe una fuerte coincidencia en que deben organizarse en módulos ( $\mathrm{f}=16$ muy de acuerdo; $\mathrm{f}=4$ de acuerdo) y que cada módulo debe tener sus propias actividades ( $\mathrm{f}=15$ muy de acuerdo; $\mathrm{f}=5$ de acuerdo), utilizando medios audiovisuales para reforzar el aprendizaje ( $\mathrm{f}=19$ muy de acuerdo; $\mathrm{f}=1$ de acuerdo) e indicando que es interesante que existan espacios para intercambiar materiales, tanto por profesor-alumno ( $\mathrm{f}=17$ muy de acuerdo) como por los propios alumnos ( $\mathrm{f}=12$ muy de acuerdo; $\mathrm{f}=8$ de acuerdo).

Los contenidos deben adaptarse al nivel y necesidades de los alumnos ( $\mathrm{f}=13$ muy de acuerdo; $\mathrm{f}=7$ de acuerdo) y debe utilizarse una metodología flexible que se adapte a las características de los destinatarios ( $\mathrm{f}=11$ muy de acuerdo; $\mathrm{f}=9$ de acuerdo).

Una serie de preguntas del cuestionario se centraban en la evaluación, y al respecto indicar que mayoritariamente están de acuerdo con que debe haber ejercicios de autoevaluación ( $\mathrm{f}=18$ muy de acuerdo), con que se debe poner en marcha la heteroevaluación ( $\mathrm{f}=10$ muy de acuerdo; $\mathrm{f}=10$ de acuerdo) y no tanto con la coevaluación ( $\mathrm{f}=7$ muy de acuerdo; $\mathrm{f}=6$ de acuerdo; $\mathrm{f}=4$ moderadamente de acuerdo; y $\mathrm{f}=3$ moderadamente en desacuerdo).

\section{- Autonomía de los estudiantes}

La mayoría piensa que los estudiantes deben tener la posibilidad de decidir diferentes itinerarios y alterar la secuencia del curso ( $\mathrm{f}=11$ de acuerdo; $\mathrm{f}=6$ muy de acuerdo; $f=3$ moderadamente de acuerdo). También se muestran de acuerdo con que el estudiante debe tener la posibilidad, en función de los conocimientos previos, la experiencia y de los recursos seleccionados, inscribirse en diferentes niveles de profundización ( $\mathrm{f}=12$ muy de acuerdo; $\mathrm{f}=8$ de acuerdo). Indicar también que son partidarios de permitir que el alumno incorpore nuevos conocimientos al entorno virtual $(\mathrm{f}=13$ muy de acuerdo; $\mathrm{f}=7$ de acuerdo).

Para finalizar, todos señalan que debe haber actividades dirigidas a aprender a aprender $(\mathrm{f}=10$ muy de acuerdo; $\mathrm{f}=10$ de acuerdo) y actividades optativas ( $\mathrm{f}=15$ muy de acuerdo; $\mathrm{f}=5$ de acuerdo).

\section{- Desventajas del $e$-learning}

Pocas las desventajas que los docentes perciben del e-learning. Así 14 se muestran en desacuerdo con que en la teleformación exista una descoordinación entre los docentes. También 16 docentes no están de acuerdo con la afirmación "En la teleformación los profesores tienen a su cargo una gran número de alumnos, dificultando el seguimiento individualizado". 12 de los encuestados se posicionan en desacuerdo respecto a los docentes tienen poca experiencia en esta modalidad formativa. 11 se encuentran moderadamente en desacuerdo y 4 en desacuerdo respecto a que supone una dificultad para los teleformadores estructurar los contenidos de forma adecuada, pedagógica y adaptada a las necesidades de la teleformación.

\section{- Diseño de las plataformas}

Por lo general los teleformadores están de acuerdo con que es necesario incorporar en la plataforma una simulación de cómo navegar $(\mathrm{f}=12$ muy de acuerdo; $f=8$ de acuerdo), así como de la necesidad de que los alumnos reciban al principio formación respecto a su utilización ( $\mathrm{f}=14$ muy de acuerdo). 
También se posicionan a favor de la afirmación "El diseño general del sitio web y la ubicación de las diferentes partes deben ser reconocibles y usables" ( $\mathrm{f}=17$ muy de acuerdo) $\mathrm{y}$ con que las aplicaciones y servicios telemáticos deben resultar de fácil manejo ( $\mathrm{f}=17$ muy de acuerdo; $\mathrm{f}=3$ de acuerdo).

\section{- Dificultad en el uso de las plataformas}

Existe desacuerdo ( $\mathrm{f}=15$ moderadamente en desacuerdo; $\mathrm{f}=5$ en desacuerdo), en que en la teleformación suele existir problemas técnicos. Posicionándose moderadamente en desacuerdo o en desacuerdo $(\mathrm{f}=13$ moderadamente en desacuerdo; $f=7$ en desacuerdo) con que uno de los problemas para su utilización es que los alumnos no saben utilizar la plataforma.

\section{- Coste de la teleformación}

Por lo que se refiere a los costes se muestran en desacuerdo $(\mathrm{f}=12)$ o moderadamente en desacuerdo $(\mathrm{f}=8)$ en que los costes de seguimiento de los alumnos son mínimos en tiempos y esfuerzos. Estando de acuerdo 11 encuestados en que la adaptación de los contenidos a la modalidad telemática supone una gran inversión económica. Aunque 12 están de acuerdo con que los costes son altos, 8 indican que son bajos.

\section{- Comparación de la formación presencial y la teleformación}

14 personas se muestran en desacuerdo y 6 moderadamente en desacuerdo, con que existe un mayor riesgo de abandono por parte de los alumnos en la teleformación que en la formación presencial. Mostrándose los profesores en desacuerdo $(\mathrm{f}=15)$ con que en la formación presencial se da un seguimiento más personalizado que en la teleformación. De todas formas, todos señalan que el estudiante es más autónomo en la teleformación que en la formación presencial ( $\mathrm{f}=13$ muy de acuerdo; $\mathrm{f}=7$ de acuerdo).

Hay diferentes opiniones con respecto a que en la teleformación se debilita la comunicación entre docente y alumnos ( $\mathrm{f}=6$ moderadamente en desacuerdo; $\mathrm{f}=5$ en desacuerdo; $\mathrm{f}=4$ de acuerdo; $\mathrm{f}=3$ moderadamente de acuerdo; $\mathrm{f}=2$ muy de acuerdo).

Al mismo tiempo los encuestados se posicionan en desacuerdo con que los docentes necesitan mayor formación didáctica para impartir formación online que para la presencial ( $\mathrm{f}=10$ en desacuerdo; 7 moderadamente en desacuerdo).
Sí hemos encontrado discrepancias en las opiniones con respecto a que la teleformación dificulta el desarrollo de habilidades sociales ( $\mathrm{f}=7$ desacuerdo; $f=7$ moderadamente en desacuerdo; $f=4$ moderadamente de acuerdo; $\mathrm{f}=2$ de acuerdo); no obstante, la mayoría están en desacuerdo o moderadamente en desacuerdo con que en la teleformación se dificulta el desarrollo de habilidades sociales.

Se muestran en desacuerdo o moderadamente en desacuerdo ( $\mathrm{f}=9$ desacuerdo; $\mathrm{f}=10$ moderadamente en desacuerdo; $\mathrm{f}=1$ moderadamente de acuerdo) cuando se señala que en la teleformación el docente tiene más volumen de trabajo que en la formación presencial.

Los resultados indican que la mayoría están en desacuerdo o moderadamente en desacuerdo $(\mathrm{f}=10$ moderadamente desacuerdo; $\mathrm{f}=7$ desacuerdo) con la opinión de que en la teleformación se desarrollan principalmente conocimientos teóricos. No obstante, hay un mínimo porcentaje que se postula de acuerdo o moderadamente de acuerdo ( $\mathrm{f}=2$ moderadamente de acuerdo; $\mathrm{f}=1$ de acuerdo).

Para finalizar, indicar que los encuestados se muestran en desacuerdo ( $\mathrm{f}=11$ en desacuerdo; $\mathrm{f}=9$ moderadamente en desacuerdo), respecto a que la formación presencial posibilita una formación más completa que la teleformación.

- Nivel formativo de las personas ocupadas que se forman por esta modalidad

10 encuestados están moderadamente de acuerdo y 6 de acuerdo, con que las personas ocupadas que se forman en teleformación son personas con estudios universitarios. Pero, encontramos una pequeña contradicción con los resultados obtenidos en el ítem que indica que "Existe el mismo número de personas que se forman por esta vía en todos los niveles formativos", ya que hay 8 que señalan estar moderadamente de acuerdo, 6 moderadamente en desacuerdo y 6 en desacuerdo; por ello, no debemos dar fiabilidad a estos resultados.

\section{CONCLUSIONES}

A la hora de presentar las conclusiones de nuestro trabajo, vamos a realizarlas de acuerdo a los diferentes objetivos que nos hemos planteado.

- Valorar el impacto de la formación de personas ocupadas desarrollada vía teleformación

Tras analizar los datos, podemos afirmar que la teleformación reduce el tiempo que las personas necesitan para llegar a ser y permanecer competentes en sus puestos de trabajo, permitiendo a los 
trabajadores actualizar su conocimiento y dar respuesta a las necesidades del entorno.

Esta modalidad formativa facilita el desarrollo personal y profesional de las personas ocupadas, ya que les aporta flexibilidad en tiempo y espacio, a la vez que reducen costes (transporte, material formativo...). Gracias a la flexibilización que aporta la teleformación, podemos decir que esta modalidad es la más oportuna para las personas ocupadas, puesto que les permite compatibilizar el horario laboral con la formación. Dicha afirmación se demuestra cuando la persona entrevistada destaca que si no existiese la teleformación la mayoría de las personas que se habían formado no hubiesen podido acceder a la formación por diversos motivos (compatibilizar horario laboral y familiar...).

Los contenidos desarrollados en teleformación son significativos y de calidad, pero tenemos que ser rigurosos con este matiz, puesto que como cualquier acción formativa necesita planificación de las acciones, de los recursos, los métodos y tener en cuenta a las personas destinatarias de la formación.

- Analizar la metodología y medios didácticos que se deben utilizar en la formación de personas ocupadas en la modalidad teleformación

De acuerdo con nuestros entrevistados, a la hora de diseñar una acción formativa impartida de forma telemática, nos debemos centrar no solo en los contenidos sino también en los destinatarios, recursos, las herramientas de comunicación, la temporalización... en definitiva, en un cúmulo de variables. Coincidiendo con ello con las propuestas que han realizado diferentes autores (Cabero, 2007; Cabero y Castaño, 2007).

Los objetivos y los destinatarios de la formación deben ser el punto de partida para el diseño de las acciones formativas. No debemos olvidar que la formación de personas ocupadas va destinada a personas adultas, con experiencia y con un bagaje de vida que debe ser aprovechado para enriquecer la formación, tanto individual como grupal.

Las personas adultas tienen una experiencia y un conocimiento del que hay que partir para diseñar la formación. Y es que, al tratarse de personas adultas, se debe tener en cuenta la experiencia que tienen, creando acciones que permitan desarrollar el conocimiento a través del dialogo y el trabajo colaborativo, de manera que se construya el conocimiento mediante la reflexión y el intercambio de conocimiento. Para este fin, se deben crear entornos de trabajo colaborativo que permitan al profesor y a los alumnos compartir materiales $\mathrm{y}$ conocimientos, a la vez que van generando y reciclando el conocimiento.

La forma de organizar los contenidos que proponen es mediante módulos estructurados que deben tener sus propios objetivos de aprendizaje, facilitando al alumno entender la finalidad última de cada contenido. Utilizando para su desarrollo una diversidad de recursos (textos, secuencias de vídeos,...). Aspecto que coincide con las propuestas que realizan diferentes autores respecto al diseño de materiales para la red (Cabero y Gisbert, 2005).

Debe haber una combinación adecuada de contenidos teóricos y prácticos. Con respecto a la parte práctica, cada módulo debe presentar sus propias actividades, siguiendo siempre una misma estructura; un ejemplo sería que todas las actividades concreten los objetivos que se quieren lograr, unas recomendaciones para hacer la actividad y una rúbrica de evaluación que permita al alumno conocer los criterios que se tendrán en cuenta para valorar la actividad. En este aspecto de la rúbrica debemos señalar que se están configurando como un instrumento significativo para la evaluación (Cabero, 2013).

Por lo que se refiere a la evaluación, son partidarios de la combinación de la autoevaluación por parte del estudiante y la heteroevaluación; sin embargo, no se mostraron tan dispuestos con la utilización de la coevaluación. Señalar, que son partidarios de hacer una evaluación previa a los estudiantes; de esta manera, el profesor adquiere información sobre el nivel de los alumnos y puede adaptar la formación.

Con respecto al tiempo, las fechas deben estar establecidas desde el principio para que los alumnos puedan organizarse. En este sentido, los tiempos deben ser flexibles.

- Analizar la autonomía que deben tener los estudiantes en la teleformación

Un elemento característico de esta modalidad formativa es la autonomía de los estudiantes. Y por ello la persona debe poseer una formación adecuada para valorar si el curso es adecuado o no para su perfil, decidir si sus conocimientos previos permiten acceder a un curso o no, así como tomar decisiones en el proceso de enseñanza-aprendizaje.

Con respecto a la autonomía del estudiante durante la formación, se le debe permitir que incorpore nuevos conocimientos al entorno virtual, ya que, como hemos indicado, son personas con experiencia y esta característica puede enriquecer el aprendizaje.

Por otra parte, según los encuestados se debe incentivar la autonomía de los alumnos; para ello, 
hay que proponer actividades optativas y actividades dirigidas a aprender a aprender, tales como: búsqueda de información, elaboración de propuestas, informes... También deben tener la posibilidad de decidir diferentes itinerarios y alterar la secuencia del curso.

\section{- Apreciar las posibles desventajas del $e$ - learning}

Señalar que pocas son las desventajas que los encuestados perciben que presenta la teleformación; así desmienten que en la teleformación exista descoordinación entre los docentes, que los profesores suelen tener poca experiencia en esta modalidad formativa y que la falta de presencialidad puede ser resuelta mediante la utilización de diferentes herramientas de comunicación sincrónicas y asincrónicas.

Sí se reclama que el número de alumnos por docente debe situarse entre 10 y 15 .

- Valorar las plataformas que se deben utilizar en la teleformación y su dificultad

Para los encuestados es importante que en la plataforma utilizada se incorpore una simulación de cómo navegar y utilizarla, ya que no siempre los alumnos conocen su uso. Aunque ello no debe evitar que los alumnos reciban una formación sobre su funcionamiento antes de comenzar el curso. También se valora como significativo que la plataforma responda siempre de la misma forma a las mismas acciones del usuario y estar siempre ubicados en el mismo lugar.

- Valorar el coste y el diseño de la formación a través de esta vía

Por lo general, los encuestados consideran que los costes de aplicación son elevados, y a los de la virtualización de los contenidos hay que incorporarles los de seguimiento de los alumnos.

- Valorar si la teleformación logra igual o mejor preparación que los cursos impartidos de forma presencial.

Por lo que se refiere al abandono del curso, los encuestados señalan que puede producirse de igual manera en la teleformación que en la formación presencial. Desarrollándose en la teleformación los mismos conocimientos teóricos y prácticos que en la formación presencial.

- Valorar si hay relación entre el nivel formativo y las personas ocupadas que se forman vía teleformación

Con respecto al nivel formativo de las personas que se forman por esta vía no podemos afirmar ni desmentir que la mayoría tengan formación universitaria, ni tampoco podemos decir que exista el mismo número de personas que se forman por esta vía en todos los niveles formativos, ya que existen diferencias de opiniones en estas cuestiones y la coordinadora no nos ha podido facilitar esos datos.

En consecuencia, podemos señalar que los encuestados perciben que la teleformación puede ser una acción válida para la formación de las personas ocupadas, si bien su éxito depende de prestarle atención a una serie de aspectos que van desde la forma de estructurar los cursos, hasta concebirlos de manera participativa y colaborativa.

\section{REFERENCIAS}

Barroso, J., y Cabero, J. (2013). La utilización del juicio de experto para la evaluación de TIC: el coeficiente de competencia experta. Bordón, 65(2), 25-38.

Belloch, C. (2013). Diseño instruccional. Universidad de Valencia: Unidad de Tecnología Educativa (UTE). http://www.uv.es/ bellochc/pedagogia/EVA4.pdf

Bisquerra, R. (Coord.) (2012). El proceso de investigación (Parte 2). En R, Bisquerra (Ed.), Metodología de la investigación educativa (pp.127-160). Madrid, España: La Muralla.

Blázquez, E.M. (2012). Estado de la cuestión. La formación como respuesta a las nuevas necesidades del mercado laboral. En E.M, Blázquez (Ed.), El sistema de formación profesional para el empleo: hacia la creación de un derecho laboral (pp.19-23). Pamplona, España: Aranzadi SA.

Cabero, J., y Barroso, J. (2013). La escuela en la sociedad de la información. La escuela 2.0. En J, Barroso y J. Cabero (Eds.), Nuevos escenarios digitales (pp.21-36). Madrid, España: Pirámide.

Cabero, J., y Gisbert, M. (2005). Materiales digitales y procesos de aprendizaje del alumno adulto. En J, Cabero y M, Gisbert (Eds.), La formación en internet. Guía para el diseño de materiales didácticos (pp.15-28). Sevilla, España: Eduforma.

Cabero, J. (2007). Elearning: variables a considerar en su diseño e incorporación a los procesos de formación. En J. Cabero y R. Romero (Eds.), Diseño y producción de TIC para la formación. Nuevas tecnologías de la información y la comunicación (pp.145-166). Barcelona, España: UOC.

Cabero, J., y Castaño, C. (2007). Bases pedagógicas del elearning. En J. Cabero y J. Barroso (Eds.), Posibilidades de la teleformación en el Espacio Europeo de Educación Superior (pp-21-45). Barcelona, España: Octaedro.

Cabero, J., y Pérez, A. (2007). Estrategias didácticas para la red: estrategias centradas en la individualización de la enseñanza, estrategias centradas en el trabajo colaborativo y estrategias para la enseñanza en grupo. En J. Cabero y J. Barroso (Eds.), Posibilidades de la teleformación en el Espacio Europeo de Educación Superior (pp.107-127). Barcelona, España: Octaedro.

Cabero, J. (2013). La formación virtual en el nuevo entramado 2.0: el e-learning 2.0. En J. Cabero y J.I, Aguaded (Eds.), Tecnologías y medios para la educación en la esociedad (pp.23-51). Madrid, España: Anaya.

Cabero, J. (dir.). (2014). Manual para el desarrollo de la formación virtual en el Instituto Tecnológico de Santo Domingo. Santo Domingo: Intec. 
Carwile, J. (2007). A Constructivist Approach to Online Teaching and Learning. Inquiry, 12(1), 68-73.

Castaño, C., Maíz, I., Palacio, G., y Villaroel, J.D. (2008). Elearning 2.0. Implicaciones en el aprendizaje. En C. Cataño., I. Maiz., G, Palacio y J.D. Villaroel (Eds.), Prácticas educativas en entornos Web 2.0 (pp.141-166). Madrid, España: Síntesis.

Conner, M. (2013). Introduction to informal learning.http://marciaconner.com/resources/\%20informa l-learning/

Cuci, R. (2014). Satisfacción de los estudiantes respecto a las acciones formativas e-learning en el ámbito universitario. Pixel-Bit. Revista de Medios y Educación, 44, 215-229.

Fandos, M., y Cano, J. (2013). Formación a distancia y retos actuales en los roles docentes y su vinculación con la empresa: propuesta y controversias. Revista Electrónica de Tecnología Educativa, 45, 1-13.

Martín, A.M., Domínguez, M., y Paralera, C. (2011). El entorno virtual: un espacio para el aprendizaje colaborativo. Edutec. Revista electrónica de tecnología educativa, 35 , 1-9.

Martín, M. A., y López, E. (2012). La sociedad de la información y la formación del profesorado. Eactividades y aprendizaje colaborativo. RIED. Revista Iberoamericana de Educación a Distancia, 15(1), 15-35.

Martínez, A.C. (2009). El diseño instruccional en la educación a distancia. Un acercamiento a los Modelos. Apertura, 9 (10), 104-119.

Monge, C. (2011). E-learning: integración de las tecnologías de la información y la comunicación en los sistemas de educación. En M.L, Sevillano (Ed.), Medios, recursos didácticos y tecnología educativa (pp.83-120).Madrid, España: Pearson.

Núñez, T.F. (2011).Entornos virtuales de aprendizaje (EVEA): Formación profesional. Edutec-e. Revista electrónica de tecnología educativa, 37, 1-15.

Rifkin, J. (2014). La sociedad de coste marginal cero. Paidós, España: Barcelona.

Rodríguez, N. (2014). Fundamentos del proceso educativo a distancia: enseñanza, aprendizaje, evaluación, RIED, 17 (2), 75-99.

Sáez, J. (2010). Informe sobre la Formación Profesional a distancia en España. Organización, oferta, metodología y herramientas utilizadas. Parte $1^{\mathrm{a}}$.Introducción y ámbito autonómico. RED, Revista de Educación a Distancia, $25,1-39$.

Salinas, J., Pérez, A., y De Benito, B. (2008). Metodologías centradas en el alumno para el aprendizaje en red. Madrid, España: Síntesis.

Shahabadi, M. y Uplane, M.(2015). Synchronous and asynchronous e-learning styles and academic performance of e-learners. Procedia-Social and Behavioral Sciences, 176, 129-138.

Tîrziu, A.M., Vrabie, C. (2015). Education 2.0: E-Learning Methods. Procedia - Social and Behavioral Sciences, 186, $376-380$.

Vallejo, D. (coord.) (2015). Empleo, personas y organizaciones. Cambios y transformaciones. Madrid, España: Pirámide.

Vidal, A., y Camarena, B. (2014). Retos y posibilidades de los cursos en línea a partir de una experiencia concreta. Pixel-Bit. Revista de Medios y Educación, 44, 19-34. 